\section{UMAVC: A program for computing comparisons in multifactor designs with unequal cell $\mathrm{Ns}$}

\author{
ALAN J. WILSON \\ Southern College of Optometry, Memphis, Tennessee 38104 \\ and \\ NERELLA V. RAMANAIAH \\ Southern Illinois University, Carbondale, Illinois 62901
}

While some computer center libraries include programs that perform comparisons for multifactor analysis of variance (ANOVA) designs, these programs are usually limited to designs with equal cell Ns. Program UMAVC extends these basic capabilities to include factorial designs with unequal cell Ns (Keppel, 1973; Myers, 1972). The program employs unweighted means techniques to assess comparisons for main effects (example: $A_{\text {comp }}$ ) and for interactions (example: $A_{\text {comp }}$ by $\mathrm{B}$ by $\mathrm{C}$ ).

Description. This program consists of two major segments. The first segment performs one-, two-, three-, or four-way unweighted means ANOVAs for standard factorial designs (fixed effect only) having either equal or unequal cell Ns; this program segment is similar in design to Veldman's (1967) AVAR23 program. The second segment performs comparisons requested by the user. These comparisons may be orthogonal or nonorthogonal, or tests for trend depending upon the coefficients that are specified.

Input. Three program control cards precede the data: an ANOVA problem identification card, an ANOVA design card, and a data format card. The data is read in accordance with the user-specified format and is arranged with the subscript of the last factor varying the fastest. Cards containing the cell Ns are placed before each "cell" of data cards. A card containing the comparison problem identification immediately follows the data. Cards specifying the desired comparisons and their coefficients complete the deck setup.

Output. Output from UMAVC consists of (1) a standard unweighted means ANOVA table including the probabilities of the computed F ratios, (2) cell means and cell Ns, and (3) a comparison table including sums of squares, degrees of freedom, mean squares and $\mathrm{F}$ ratios accompanied by their associated probabilities.

Restrictions. The number of levels of each ANOVA factor must not exceed 10 . The program can analyze only one comparison per factor in a given run; different comparisons for different factors may be analyzed in a single run. In the case of trend comparisons, for example, to investigate both linear and quadratic trends for factor $\mathrm{A}$ would require two runs, while a linear trend for factor $A$, a quadratic trend for factor $B$, a cubic trend for factor $C$, and a quartic trend for factor $D$ could be investigated in a single run.

Computer and language. The program is written in FORTRAN IV and was developed on an IBM 370/155 with virtual memory. It executes in approximately 192K of core; reducing the maximum number of levels for the ANOVA factors below the present value of 10 would greatly reduce the program's core requirements. Compilation time is approximately $30 \mathrm{sec}$. Data sets of average size may be analyzed in 5-10 sec of CPU time. Two direct access devices are required. The program consists of a mainline and 13 subroutines.

Availability. A documented listing of UMAVC is available at no cost from the first author at: Southern College of Optometry, 1245 Madison Avenue, Memphis, Tennessee 38104.

\section{REFERENCES}

KEPPEL, G. Design and analysis: A researcher's handbook. Englewood Cliffs, N.J : Prentice-Hall, 1973.

MYERS, J. L. Fundamentals of experimental design (2nd ed.). Boston: Allyn and Bacon, 1972.

VELDMAN, D. FORTRAN programming for the behavioral sciences. New York: Holt, Rinehart and Winston, 1967. 